\title{
GMR
}

\section{Influence of various quantitative trait loci (QTL) mapping methods on the mapping accuracy under varying heritability levels}

\author{
C.F. Su, Z.B. Liu and Y.G. Li \\ Department of Life Sciences, Liupanshui Normal University, Liupanshui, \\ Guizhou, China \\ Corresponding author: C.F. Su \\ E-mail: chfsu2008@163.com \\ Genet. Mol. Res. 14 (4): 13003-13012 (2015) \\ Received April 23, 2015 \\ Accepted July 2, 2015 \\ Published October 21, 2015 \\ DOI http://dx.doi.org/10.4238/2015.October.21.21
}

ABSTRACT. The study of quantitative trait effects is of great significance for molecular marker-assisted breeding. The accuracy of quantitative trait loci (QTL) mapping is the key factor affecting marker-assisted breeding, and is extremely significant. The effect of different heritability rates (10, $30,50,70$, and $90 \%$ ) on the accuracy of QTL mapping of five recombinant inbred lines (RILs) were analyzed via computer simulation. RILs display additive and epistatic genetic effects. The QTLs were analyzed using four different mapping procedures: multiple QTL model (MQM), composite interval mapping (CIM), multiple interval mapping (MIMR), and inclusive composite interval mapping (ICIM). The results revealed an increase in the QTL mapping accuracy and QTL detection power, and a decrease in the QTL interval range with the increase in heritability; conversely, an irregular number of false positive QTLs were generated. CIM and MQM only screen the additive and dominant effects; MIMR and ICIM screen the additive, dominant, and epistatic effects. The highest QTL detection power obtained using MQM and CIM was only $75 \%$, while MIMR and ICIM showed a detection power of $100 \%$. At heritability rates of more than 50 and less than $10 \%$, the detection powers of the MIMR and ICIM procedures were $>95$ 
and $<35 \%$, respectively. QTL mapping has no significance at heritability rates $<10 \%$. The results of this study suggest that QTL mapping has significance at a heritability rate $>30 \%$ (at least $>10 \%$ ) for practical marker-assisted breeding.

Key words: QTL mapping; Heritability; Multiple procedures; Accuracy; Detection power; Computer simulation

\section{INTRODUCTION}

The late nineteenth century was witness to a rapid development in genetic linkage maps, QTL mapping methods, and related research on molecular genetics, accompanying the advancement in molecular marker technology. This has provided breeders with a powerful tool to study the genetic mechanism of quantitative traits of DNA at a molecular level. The mapping of quantitative trait loci (QTL) in plants and animals has become a worldwide phenomenon. A previous study has reported the mapping of 1287, 630, and 657 QTLs in pigs, cattle, and chicken (among animals), respectively, up to August, 2006 (Hu et al., 2007). Among plants, over 2000 QTLs have been reported (mapped) in soybean up to 2010 (Su et al., 2010a). QTLs can be used with high accuracy in markerassisted breeding or map-based cloning; however, false-positive QTLs cannot be utilized for these applications. Although QTL mapping has developed quickly over the past few years, a large number of these mapped QTLs cannot be used in marker-assisted breeding because of their low accuracy and dependability. The results of QTL mapping performed repeatedly (several times) have always been different (Beavis, 1994, 1998). Based on its accuracy, a genetic linkage map could be used for further marker-assisted breeding. The accuracy of QTL mapping is dependent on the detection power, the identified QTL range, and accuracy of the genetic parameters. Effective strategies must be developed and analyzed to increase the accuracy and dependability of QTL mapping; this is especially of significance in the case of marker-assisted breeding in plants.

Several QTL mapping methods, such as interval mapping (IM), composite interval mapping (CIM), and multiple interval mapping (MIM), have been developed and improved gradually over time. Mapmaker/QTL is the earliest QTL analyzing software, reported for the first time by Lander and Bostein (1989). Mapmaker/QTL is based on the IM model. Since then, other QTL mapping software, such as QTL Cartographer (Basten et al., 1994), PLABQTL (Utz and Melchinger, 1996), Map Manager (Manly et al., 2001), QGene (Nelson, 1997), MapQTL (van Ooijen and Maliepaard, 1996), PGRI (Lu and Liu, 1995), QTLMAPPER (Wang et al., 2003), QTLSTA, IciMapping (Li et al., 2007), and QTLnetwork (Yang et al., 2007, 2008) have been developed and reported. A number of QTL mapping software or software packages can be downloaded freely from a website created by the University of Wisconsin-Madison (http://www.stat.wisc.edu/ yandell/qtl/software/) (Su et al., 2013). The composite interval mapping (CIM) and multiple interval mapping (MIM) functions of the WinQTL Cartographer v.2.5 (Wang et al., 2007) software program, the multiple-QTL model (MQM) of the MapQTL v.5.0 (van Ooijen et al., 2004) program, and the inclusive composite interval mapping (ICIM) model of the IciMapping v.2.0 software platform (Li et al., 2007) are four types of QTL mapping models that have found worldwide applicability.

The statistical algorithms and genetic models of the six mapping procedures are different (Utz and Melchinger, 1996; van Ooijen and Maliepaard, 1996; Kao et al., 1999; Wang, 2009). The CIM and MQM models only estimate the additive and dominant characteristics, while MIM and ICIM can be used to estimate the additive, dominant, and epistasis characteristics of the genetic models. In a practical experiment where the genetic model of the data is unknown, a multiple model 
mapping strategy must be utilized (Su et al., 2013).

The heritability and marker density of the linkage map are two main factors that significantly affect the QTL mapping accuracy. Over the past few years, several researchers have attempted to analyze the QTL mapping accuracy, in order to increase the efficiency of marker-assisted breeding. The results obtained varied for different researchers because of the differences in the simulation methods, segregation population, and QTL mapping procedures used. A majority of the previous studies on QTL mapping accuracy consider only the additive and dominant effects, and not the epistatic effects.

In this study, five RIL populations with different levels of heritability $(10,30,50,70$, and $90 \%)$ were subjected to computerized simulation and (subsequently) QTL analysis. The RIL populations displayed the same genetic model $\left(y=\mu+a_{1}+a_{2}+a a_{12}+a_{3}+e\right)$. The influence of the different levels of heritability on the accuracy of QTL mapping was analyzed in this study. The results of this study could serve as a theoretical and practical basis for future marker-assisted breeding in plants.

\section{MATERIAL AND METHODS}

\section{Genetic model setting and analysis index}

Five sets of RIL data on the additive and interactive functions between genes in a genetic model were produced (simulated 120 times) based on the differences in the heritability level. The five sets of RIL data, named RIL-1 RIL-5, were completed by computer simulation, and utilized for further QTL detection. A sample size of 250 family lines was set for each simulated RIL. Three QTLs located within three linkage groups, with the genetic model " $y=\mu+a_{1}+a_{2}+a a_{12}+a_{3}+$ $e$ ", were set up. The letter $y$ indicates the phenotypic value of quantitative characters, while the letters $\mu$ and $a$ represent the general average number and mean additive effects, respectively. The combined aa indicates the interaction between the genes (epistatic effect), while the letter $e$ represents the error that complies with the normal distribution "e $\sim \mathrm{N}\left(0, \sigma_{e}^{2}\right)$ ".

The analysis index calculated for all 120 simulations considered the QTL detection power, number of false-positive QTLs, variation range of the effects of QTLs, accuracy of the QTL effects, coefficient of variation (CV) of the QTL effects, and the variation range of QTL position. The accuracy of the QTL effects was calculated using the following equation "1-|average value-set value| / set value". The QTLs identified within $10 \mathrm{cM}$ (centimorgan) were considered to be identical to the QTL analyzed in this study.

\section{RIL simulation}

\section{Marker genotype and QTL simulation}

The computer simulation methodology reported by He et al. (2001) was utilized as the reference for this study. This method assumed the presence of two adjacent markers, $M_{k}$ and $M_{k+1}$, on a chromosome with a recombination frequency $r$. The genotypes of the two markers $\left(M_{k}\right.$ and $\left.M_{k+1}\right)$ were recorded to be $x_{k}$ and $x_{k+1}$. The values assigned to $x_{k}$ and $x_{k+1}(0$ and 1 , respectively) indicate that the DNA of the two loci arise from two homozygous parents (P1 and P2, respectively). While the value of $x_{k}$ was confirmed to be " 1 ", the genotype of $x_{k+1}$ was inferred and confirmed from the known genotype of $x_{k}$. The presence of $n_{1}$ individuals with the genotype $x_{k}$ (with a value of " 1 ") was assumed. A total of $n_{1}$ uniformly distributed random numbers (between 0 and 1 ) were generated continuously, and recorded as " $r n d$ ". The value of $x_{k+1}$ was determined to be " 1 ", when 
$r n d \leq(1-r)^{2}$. The value of $x_{k+1}$ was determined to be " 0 " when $r$ d $>(1-r)^{2}$. The value of $x_{k+2}, x_{k+3}$, and so on, were inferred and confirmed based on the known value of $x_{k+1}$. The value of $x_{k}$ was confirmed to be " 0 "; the values of $x_{k+1}, x_{k+2}, x_{k+3}$, and so on were inferred based on an assumed $x_{k}$ value of " 1 ".

\section{Confirmation of the marker genotypes at the starting position of the simulated linkage group}

The $x_{k}$ value can be determined as 0 or 1 based on a probability of 0.50 . A total of $n$ random numbers were generated continuously between 0 and 1 . The value of $x_{k}$ was calculated to be 0 and 1 when the rnd values were $\leq 0.5$ or $>0.50$, respectively. The same method was applied to confirm the QTL marker genotype; this value was recorded as $x_{Q}$. Each simulated RIL generated a total of three linkage groups (Lg1, Lg2, and Lg3) containing 20 markers; these markers were used for further QTL analyses. Different recombination frequencies were set up between the two adjacent markers in the different linkage groups (0.06 in Lg1, 0.08 in Lg2, and 0.10 in Lg3). Three related $Q T L s\left(Q_{\text {set1 }}, Q_{\text {set2 }}\right.$, and $\left.Q_{\text {set3 }}\right)$ were simulated according to the different genetic models of the simulated RILs. $Q_{\text {set1 }}$ was set up to be located at the marker $12 \sim$ marker 13 (M12 M13) interval in linkage group $1(\operatorname{Lg} 1)$. On the other hand, $Q_{\text {set2 }}$ and $Q_{\text {set3 }}$ were set up to be located at the $M 12 \sim$ M13 and M6 M7 intervals in Lg2 and Lg3, respectively.

\section{Confirmation of the phenotypic value of the quantity trait}

The phenotypic values were set up according to the genetic model " $y=\mu+a_{1}+a_{2}+a a_{12}$ $+a_{3}+e$ ". The genetic parameters in the model were set up as follows: the genetic effect of $a_{1}, a_{2}$, $a_{3}$, and $a a_{12}$ were $-3,-2.5,-1.5$, and 2 , respectively. Different heritability levels $(10,30,50,70$, and $90 \%$ ) were set up for the five sets of simulated RILs.

\section{QTL mapping procedures}

Four types of QTL mapping procedures were utilized for further QTL analysis of the simulated data. These were the composite interval mapping (CIM) and regression forward selection of multiple interval mapping (MIMR) models provided in the WinQTL Cartographer v.2.5 software package, inclusive composite interval mapping (ICIM) function of the IciMapping (v.2.0) package, and the multiple-QTL model (MQM) provided by the MapQTL v.5.0 software platform. The data was analyzed up to one thousand permutations to determine the threshold logarithm of Odd's ratio (LOD) values.

\section{RESULTS}

\section{Coincidence analysis between the actual and preset values when analyzed using different procedures}

Different mapping procedures were applied to analyze the effects of QTL on five sets of RIL data (repeated 120 times). Several parameters, including the average, accuracy, minimum value, maximum value, and coefficient of variation (CV), were obtained by calculating and analyzing the 120 values obtained from the 120 simulations. The related results of all statistical analyses conducted on the QTL mapping power, the range of variation in the QTL position, and the numbers of false positive QTLs, are summarized in Table 1. 
Table 1. Estimates of genetic parameters using simulation data of five sets of recombinant inbred line (RILs) subjected to four different quantitative trait loci (QTL) mapping procedures: multiple QTL model (MQM), composite interval mapping (CIM), inclusive CIM (ICIM), and multiple interval mapping (MIMR)).

\begin{tabular}{|c|c|c|c|c|c|c|c|c|c|c|}
\hline Procedure & Effect & True value & Average & Accuracy & Minimum & Maximum & CV & RanQTL (cM) & Power (\%) & False QTL \\
\hline \multicolumn{11}{|c|}{ MQM, 120 times of simulation } \\
\hline \multirow[t]{4}{*}{$10 \%$} & a1 & -3.00 & -3.62 & 0.28 & -5.16 & -2.98 & 0.130 & 24.59 & 20 & - \\
\hline & a2 & -2.50 & -3.78 & 0.49 & -5.00 & -2.94 & 0.140 & 39.08 & & - \\
\hline & aa12 & 2.00 & - & - & - & - & - & - & & - \\
\hline & a3 & -1.50 & -3.58 & 0.28 & -4.56 & -3.12 & 0.148 & 24.20 & & - \\
\hline \multirow[t]{4}{*}{$30 \%$} & a1 & -3.00 & -2.84 & 0.95 & -4.15 & -1.78 & 0.160 & 11.46 & 51 & - \\
\hline & a2 & -2.50 & -2.46 & 0.99 & -3.38 & -1.77 & 0.149 & 12.42 & & - \\
\hline & aa12 & 2.00 & - & - & - & - & - & - & & - \\
\hline & a3 & -1.50 & -2.11 & 0.60 & -2.63 & -1.79 & 0.126 & 32.45 & & - \\
\hline \multirow[t]{4}{*}{$50 \%$} & a1 & -3.00 & -2.75 & 0.92 & -3.51 & -1.89 & 0.106 & 0.34 & 58 & - \\
\hline & a2 & -2.50 & -2.39 & 0.96 & -3.18 & -1.76 & 0.121 & 0.00 & & - \\
\hline & aa12 & 2.00 & - & - & - & - & - & - & & - \\
\hline & a3 & -1.50 & -1.63 & 0.91 & -2.12 & -1.33 & 0.115 & 38.9 & & - \\
\hline \multirow[t]{4}{*}{$70 \%$} & $\mathrm{a} 1$ & -3.00 & -2.81 & 0.94 & -3.26 & -2.14 & 0.070 & 0.00 & 65 & - \\
\hline & a2 & -2.50 & -2.37 & 0.95 & -2.97 & -1.86 & 0.081 & 0.00 & & - \\
\hline & aa12 & 2.00 & - & - & - & - & - & - & & - \\
\hline & a3 & -1.50 & -1.33 & 0.89 & -1.75 & -1.11 & 0.108 & 21.13 & & - \\
\hline \multirow[t]{4}{*}{$90 \%$} & $\mathrm{a} 1$ & -3.00 & -2.79 & 0.93 & -3.04 & -2.55 & 0.034 & 0.00 & 74 & - \\
\hline & a2 & -2.50 & -2.36 & 0.97 & -2.58 & -2.07 & 0.044 & 0.00 & & - \\
\hline & aa12 & 2.00 & - & - & - & - & - & - & & - \\
\hline & a3 & -1.50 & -1.19 & 0.79 & -1.37 & -0.97 & 0.074 & 0.39 & & - \\
\hline \multicolumn{11}{|c|}{ CIM, 120 times of simulation } \\
\hline \multirow[t]{4}{*}{$10 \%$} & a1 & -3.0 & -4.11 & 0.63 & -5.80 & -2.88 & 0.208 & 28.52 & 27.1 & - \\
\hline & a2 & -2.5 & -3.98 & 0.41 & -6.55 & -2.93 & 0.182 & 47.15 & & - \\
\hline & aa12 & 2.0 & - & - & - & - & - & - & & - \\
\hline & a3 & -1.5 & -3.96 & 0.39 & -5.56 & -2.99 & 0.172 & 29.95 & & - \\
\hline \multirow[t]{4}{*}{$30 \%$} & $\mathrm{a} 1$ & -3.0 & -2.98 & 0.99 & -4.50 & -1.75 & 0.191 & 12.46 & 62.3 & - \\
\hline & a2 & -2.5 & -2.59 & 0.96 & -3.70 & -1.55 & 0.170 & 11.42 & & - \\
\hline & aa12 & 2.0 & - & - & - & - & - & - & & - \\
\hline & a3 & -1.5 & -2.12 & 0.58 & -3.31 & -1.53 & 0.218 & 33.45 & & - \\
\hline \multirow[t]{4}{*}{$50 \%$} & a1 & -3.0 & -2.77 & 0.92 & -4.00 & -1.38 & 0.145 & 3.87 & 72 & - \\
\hline & a2 & -2.5 & -2.56 & 0.98 & -3.46 & -1.75 & 0.131 & 1.87 & & - \\
\hline & aa12 & 2.0 & - & - & - & - & - & - & & - \\
\hline & a3 & -1.5 & -1.65 & 0.90 & -2.83 & -1.12 & 0.218 & 31.56 & & - \\
\hline \multirow[t]{4}{*}{$70 \%$} & a1 & -3.0 & -2.83 & 0.94 & -3.41 & -2.12 & 0.081 & 0.34 & 75 & - \\
\hline & a2 & -2.5 & -2.52 & 0.99 & -3.33 & -2.07 & 0.085 & 0 & & - \\
\hline & aa12 & 2.0 & - & - & - & - & - & - & & - \\
\hline & a3 & -1.5 & -1.51 & 0.99 & -2.09 & -1.04 & 0.148 & 9.99 & & - \\
\hline \multirow[t]{4}{*}{$90 \%$} & a1 & -3.0 & -2.79 & 0.93 & -3.14 & -2.52 & 0.040 & 0.34 & 75 & - \\
\hline & a2 & -2.5 & -2.49 & 1.00 & -2.80 & -2.17 & 0.049 & 0 & & - \\
\hline & aa12 & 2.0 & - & - & - & - & - & - & & - \\
\hline & a3 & -1.5 & -1.48 & 0.99 & -1.82 & -1.13 & 0.088 & 1.39 & & - \\
\hline \multicolumn{11}{|c|}{ ICIM, 120 times of simulation } \\
\hline \multirow[t]{4}{*}{$10 \%$} & a1 & -3.00 & -3.89 & 0.70 & -6.35 & -2.72 & 0.198 & 30.00 & 26 & $20(A)+2(A A)$ \\
\hline & a2 & -2.50 & -3.80 & 0.48 & -5.43 & -2.75 & 0.151 & 45.00 & & \\
\hline & aa12 & 2.00 & 5.11 & 0.34 & 4.28 & 6.07 & 0.116 & - & & \\
\hline & a3 & -1.50 & -3.71 & 0.32 & -4.34 & -3.10 & 0.092 & 33.00 & & \\
\hline $30 \%$ & a1 & -3.00 & -2.90 & 0.97 & -4.95 & -1.73 & 0.198 & 1.00 & 70 & $18(\mathrm{~A})+3(\mathrm{AA})$ \\
\hline & a2 & -2.50 & -2.59 & 0.97 & 3.84 & -1.62 & 0.166 & 0.00 & & \\
\hline & aa12 & 2.00 & 2.55 & 0.73 & 2.01 & 3.27 & 0.119 & - & & \\
\hline & a3 & -1.50 & -2.03 & 0.64 & -3.23 & -1.57 & 0.162 & 35.00 & & \\
\hline $50 \%$ & a1 & -3.00 & -2.9 & 0.97 & -4.01 & -1.60 & 0.140 & 1.00 & 95 & $31(\mathrm{~A})+5(\mathrm{AA})$ \\
\hline & a2 & -2.50 & -2.54 & 0.99 & -3.32 & -1.91 & 0.122 & 1.00 & & \\
\hline & aa12 & 2.00 & 1.91 & 0.96 & 1.37 & 2.72 & 0.152 & - & & \\
\hline & a3 & -1.50 & -1.64 & 0.91 & -2.55 & -1.10 & 0.178 & 40.00 & & \\
\hline $70 \%$ & a1 & -3.00 & -2.94 & 0.98 & -3.60 & -2.38 & 0.088 & 1.00 & 98 & $27(\mathrm{~A})+5(\mathrm{AA})$ \\
\hline & a2 & -2.50 & -2.55 & 0.98 & -3.36 & -2.1 & 0.083 & 0.00 & & \\
\hline & aa12 & 2.00 & 1.89 & 0.95 & 1.44 & 2.44 & 0.108 & - & & \\
\hline & a3 & -1.50 & -1.55 & 0.97 & -2.00 & -0.98 & 0.148 & 0.00 & & \\
\hline
\end{tabular}

Continued on next page 


\begin{tabular}{|c|c|c|c|c|c|c|c|c|c|c|}
\hline Procedure & Effect & True value & Average & Accuracy & Minimum & Maximum & $\mathrm{CV}$ & RanQTL (cM) & Power (\%) & False QTL \\
\hline \multirow[t]{4}{*}{$90 \%$} & a1 & -3.00 & -2.89 & 0.96 & -2.52 & -3.49 & 0.069 & 0.00 & 100 & $23(\mathrm{~A})+7(\mathrm{AA})$ \\
\hline & a2 & -2.50 & -2.50 & 1.00 & -2.79 & -2.21 & 0.045 & 0.00 & & \\
\hline & aa12 & 2.00 & 1.92 & 0.96 & 1.49 & 2.36 & 0.069 & - & & \\
\hline & a3 & -1.50 & -1.54 & 0.97 & -1.80 & -1.16 & 0.074 & 0.00 & & \\
\hline \multicolumn{11}{|c|}{ MIMR, 120 times of simulation } \\
\hline \multirow[t]{4}{*}{$10 \%$} & a1 & -3.00 & -4.26 & 0.58 & -12.02 & -2.91 & 0.327 & 29.52 & 32.1 & $16(\mathrm{~A})+1(\mathrm{AA})$ \\
\hline & $\mathrm{a} 2$ & -2.50 & -4.10 & 0.36 & -10.40 & -2.93 & 0.277 & 44.67 & & \\
\hline & aa12 & 2.00 & 3.73 & 0.13 & 3.27 & 4.25 & 0.098 & - & & \\
\hline & a3 & -1.50 & -4.61 & 0.52 & -15.3 & -2.99 & 0.556 & 28.16 & & \\
\hline \multirow[t]{4}{*}{$30 \%$} & a1 & -3.00 & -3.15 & 0.95 & -7.73 & -1.97 & 0.236 & 9.00 & 85.4 & $12(\mathrm{~A})+1(\mathrm{AA})$ \\
\hline & a2 & -2.50 & -2.63 & 0.95 & -4.00 & -1.63 & 0.166 & 5.00 & & \\
\hline & aa12 & 2.00 & 2.10 & 0.95 & 1.53 & 2.97 & 0.166 & - & & \\
\hline & a3 & -1.50 & -2.07 & 0.62 & -4.22 & -1.46 & 0.207 & 5.51 & & \\
\hline \multirow[t]{4}{*}{$50 \%$} & a1 & -3.00 & -3.00 & 1.00 & -4.01 & -1.89 & 0.136 & 0.43 & 95.6 & $23(\mathrm{~A})+5(\mathrm{AA})$ \\
\hline & a2 & -2.50 & -2.62 & 0.95 & -3.71 & -1.77 & 0.131 & 1.00 & & \\
\hline & aa12 & 2.00 & 1.92 & 0.96 & 1.19 & 2.73 & 0.173 & - & & \\
\hline & a3 & -1.50 & -1.64 & 0.90 & -2.38 & -1.07 & 0.203 & 35.45 & & \\
\hline \multirow[t]{4}{*}{$70 \%$} & a1 & -3.00 & -3.06 & 0.98 & -3.93 & -2.56 & 0.073 & 0.09 & 100 & $13(\mathrm{~A})+1(\mathrm{AA})$ \\
\hline & a2 & -2.50 & -2.57 & 0.97 & -3.37 & -2.01 & 0.089 & 0.09 & & \\
\hline & aa12 & 2.00 & 1.92 & 0.96 & 1.30 & 2.46 & 0.106 & - & & \\
\hline & a3 & -1.50 & -1.55 & 0.97 & -2.02 & -1.05 & 0.143 & 1.39 & & \\
\hline \multirow[t]{4}{*}{$90 \%$} & a1 & -3.00 & -2.98 & 0.99 & -3.29 & -2.69 & 0.040 & 0.09 & 100 & $14(\mathrm{~A})+2(\mathrm{AA})$ \\
\hline & $\mathrm{a} 2$ & -2.50 & -2.57 & 0.97 & -5.46 & -2.24 & 0.113 & 0.09 & & \\
\hline & aa12 & 2.00 & 1.94 & 0.97 & 1.59 & 2.21 & 0.056 & - & & \\
\hline & a3 & -1.50 & -1.56 & 0.96 & -1.86 & -1.30 & 0.065 & 0.48 & & \\
\hline
\end{tabular}

Accuracy is determined as (1-|average-true valuel / true value); CV, coefficient of variation; A, additive QTL; AA, epistatic QTL pair; RanQTL, the range of QTL location.

\section{QTL detection power}

The CIM and MQM procedures could not detect the epistatic effect. The detection power of CIM and MQM was observed to be much lower than that of MIMR and ICIM; however, the heritability level was the same in all four procedures. The detection power increased with the increase in the heritability levels; the progressive increase in heritability levels from 10 to $90 \%$ resulted in a corresponding increase in the detection power of MQM from 20 to $74 \%$, of CIM from 27.1 to $75 \%$, of ICIM from 26 to $100 \%$, and that of MIMR from 32.1 to $100 \%$. The highest detection power of the MQM and CIM procedures (which can only estimate the additive and dominant effects) was $75 \%$ despite the increase in heritability. The highest detection power of MIMR and ICIM (which estimates the additive, dominant, and epistatic effects), on the other hand, was $100 \%$. Unlike MQM and CIM, the detection power of MIMR and ICIM could be increased up to $100 \%$ by increasing the heritability level.

\section{Variation range of QTL position}

The QTLs detected within $10 \mathrm{cM}$ were considered to be the same as the QTL analyzed in this study. The QTL position of the five sets of RILs, detected using different procedures, varied from 0 to $45 \mathrm{cM}$. The variation range of the QTL position showed a gradual decrease with the progressive increase in heritability levels from 10 to $90 \%$; that is, the variation range of the QTL position was at its maximum at a heritability of $10 \%$. The variation ranges of the $Q_{\text {set } 1}, Q_{\text {set2 } 2}$, and $Q_{\text {set3 }}$ positions as determined by the four different QTL mapping procedures, MQM, CIM, ICIM, and MIMR, at a heritability level of $10 \%$ were $24.59,28.52,30.00$, and $29.52 \mathrm{cM}, 39.08,47.15,45.00$, 
and $44.67 \mathrm{cM}$, and $24.20,29.95,33.00$, and $28.16 \mathrm{cM}$, respectively. The minimum variation range of the QTL position was observed at a heritability of $90 \%$. The variation ranges of the $Q_{\text {set1 }}, Q_{\text {set2 }}$, and $Q_{\text {set3 }}$ positions as determined by the MQM, CIM, ICIM, and MIMR mapping procedures, were $0.00,0.34$, 0.00 , and $0.09 \mathrm{cM}, 0.00,0.00,0.00$, and $0.09 \mathrm{cM}$, and $0.39,1.39,0.00$, and $0.48 \mathrm{cM}$, respectively.

\section{Conformity analysis between the actual and preset values}

The actual values calculated using the different procedures and the preset values were consistent unless the mapping procedure was not fit for the genetic model data; for example, the actual value of epistasis between $Q_{\text {set } 1}$ and $Q_{\text {set2 }}\left(a a_{12}\right)$ could not be detected using the CIM and MQM procedures (which cannot determine the epistasis effects; Table 1). The genetic effects, accuracy, and CV of the same QTL fluctuated to a certain extent when subjected to 120 simulations. The accuracy of $a_{1}, a_{2}$, and $a_{3}$ in RIL-1, using the MQM, CIM, ICIM, and MIMR QTR mapping procedures, were $0.28,0.30,0.70$, and $0.58,0.49,0.41,0.48$, and 0.36 , and $0.28,0.39,0.32$, and 0.52 , respectively, in RIL-1. The accuracy of $a a_{12}$, as determined by ICIM and MIMR, was 0.34 and 0.13 , respectively. The highest accuracy $(0.70)$ of the actual value of $a_{1}$ was obtained using ICIM. The least variation range of the actual value of $a_{1}$ was obtained using the MQM procedure (2.18), followed by CIM (2.92). The highest accuracy of 0.49 was obtained for the actual value of $a_{2}$, when evaluated with MQM. The least variation range was obtained when the actual value of $a_{2}$ was determined using MQM (2.06) and ICIM (2.68). On the other hand, the actual value of $a_{3}$ was calculated with highest accuracy $(0.52)$ using the MIMR procedure. The least variation range in the actual value of $a_{3}$ was obtained when determined using the MIMR and MQM procedures (1.24 and 1.44, respectively). The accuracy of $a_{1}, a_{2}$, and $a_{3}$, as determined by the MQM, CIM, ICIM, and MIMR QTL mapping procedures, were 0.95, 0.99, 0.97, and 0.95, 0.99, 0.96, 0.97, and 0.95 , and $0.60,0.58,0.64$, and 0.62 , respectively, in RIL-2. ICIM and MIMR effected accuracies of 0.73 and 0.95 to $a a_{12}$, respectively. The actual values of $a_{1}, a_{2}$, and $a_{3}$ were determined with highest accuracy $(0.99,0.99$, and 0.64) using the CIM, MQM, and ICIM models, respectively. The least variation ranges of the actual values of $a_{1}, a_{2}$, and $a_{3}$ were obtained using MQM and CIM (2.37 and 2.75), MQM and CIM (1.61 and 2.15), and MQM and ICIM (0.84 and 1.66), respectively. The accuracies of $a_{1}, a_{2}$, and $a_{3}$ in RIL-3 were determined by the MQM, CIM, ICIM, and MIMR mapping methods to be 0.92, 0.92, 0.97, and 1.00, 0.96, 0.98, 0.99, and 0.95, and 0.91, 0.90, 0.91, and 0.90 , respectively. The accuracy of $a a_{12}$ was determined to be 0.96 by both ICIM and MIMR. The actual values of $a_{1}, a_{2}$, and $a_{3}$ were determined with highest accuracy $(1.00,0.99$, and 0.91$)$ using the MIMR, ICIM, and both MQM and ICIM procedures, respectively. The least variation ranges of the actual values of $a_{1}, a_{2}$, and $a_{3}$ were obtained using MQM and MIMR (1.62 and 2.12), ICIM and MQM (1.41 and 1.42), and MQM and MIMR (0.79 and 1.31), respectively. The MQM, CIM, ICIM, and MIMR models generated $a_{1}, a_{2}$, and $a_{3}$ values in RIL-4 with accuracies of $0.94,0.94,0.98$, and $0.98,0.95,0.99,0.98$, and 0.97 , and $0.89,0.99,0.97$, and 0.96 , respectively, while ICIM and MIMR generated $a a_{12}$ values with accuracies of 0.95 and 0.96 , respectively. The actual values of $a_{1}, a_{2}$, and $a_{3}$ were determined with highest accuracy $(0.98,0.99$, and 0.99$)$ using the ICIM and MIMR, $\mathrm{CIM}$, and CIM procedures, respectively. The least variation ranges of the actual values of $a_{1}, a_{2}$, and $a_{3}$ were obtained using MQM and ICIM (1.12 and 2.12), MQM (1.11), and MQM and MIMR (0.64 and 0.97), respectively. The MQM, CIM, ICIM, and MIMR QTL mapping models generated $a_{1}, a_{2}$, and $a_{3}$ values in RIL-5 with accuracies of $0.93,0.93,0.96$, and 0.99 , respectively, 0.97, 1.00 , 1.00 , and 0.97 , respectively, and $0.79,0.99,0.97$, and 0.96 , respectively, while ICIM and MIMR generated $a a_{12}$ values with accuracies of 0.97 and 0.96 , respectively. The actual values of $a_{1}, a_{2}$, 
and $a_{3}$ were determined with highest accuracy $(0.99,1.00$, and 0.99$)$ using the MIMR, CIM and ICIM, and CIM procedures, respectively. The least variation ranges of the actual values of $a_{1}, a_{2}$, and $a_{3}$ were obtained using the MQM and MIMR (0.49 and 0.60), MQM and ICIM (0.51 and 0.58), and MQM and MIMR (0.40 and 0.56) models, respectively.

\section{Analysis of false-positive QTLs}

The counting of false positive QTLs screened by MQM and CIM is of no significance, as these two QTL mapping procedures cannot detect the epistatic effects. Therefore, only the false positive QTL numbers generated by ICIM and MIMR were counted; the statistical results are summarized in Table 1.

The number of false positive QTLs detected by ICIM and MIMR were different when the same RIL data was simulated 120 times. The ICIM and MIMR procedures detected 20 and 16 additive and 2 and 1 pair(s) of epistatic false-positive QTLs, respectively, in RIL-1. ICIM generated 18 additive and 3 pairs of epistatic false-positive QTLs, while MIMR detected 12 additive and 1 pair of epistatic false-positive QTLs in RIL-2. On the other hand, the ICIM and MIMR procedures generated 31 and 23 additive false-positive QTLs and 5 pairs each of epistatic false-positive QTLs, respectively, in RIL-3. ICIM detected 27 additive and 5 pairs of epistatic false-positive QTLs and MIMR generated 13 additive and 1 pair of epistatic false-positive QTL in RIL-4. Finally, the ICIM and MIMR procedures detected 23 and 14 additive and 7 and 2 pairs of epistatic false-positive QTLs, respectively, in RIL-5.

In summary, the detection power of QTL was observed to increase with the increase in heritability level. The highest detection power of the MQM and CIM procedures (which cannot detect the epistatic effects of five RILs) was $75 \%$, while MIMR and ICIM effected detection powers of $100 \%$. The power of detection of the four QTL mapping procedures was less than $35 \%$ at QTL heritability rates of less than $10 \%$; this can be classified as a small probability event. At QTL heritability rates of $>30 \%$, the detection power of ICIM and MIMR was $>70$ and $85 \%$, respectively. On the other hand, at a QTL heritability of $>50 \%$, the detection power of the ICIM and MIMR QTL mapping procedures was > 95\% each. The number of false-positive QTLs generated in the five sets of simulated RILs using the same QTL mapping procedure remained unchanged. Overall, the number of false-positive QTLs detected by MIMR was lesser than that detected by ICIM for the same genetic model with the same heritability. The accuracy of QTL mapping was increased by adding the heritability level. The accuracy of both $a_{1}$ and $a_{2}$, as detected by the four QTL mapping procedures was $>90 \%$ at a QTL heritability rate of $>30 \%$. The accuracy of $a_{1}, a_{2}, a_{3}$, and $a a_{12}$, as determined by the four QTL mapping procedures, was $>90 \%$ percent at QTL heritability rates of $>50 \%$. The range of variation of the QTL position, on the other hand, became narrower with the addition of the QTL heritability level. The variation ranges of both the $Q_{\text {set1 }}$ and $Q_{\text {set2 }}$ positions were $<10 \mathrm{cM}$, when calculated using the by four QTL mapping procedures, at heritability rates of > $30 \%$. Conversely, the variation in the range of $Q_{\text {set } 1}$ and $Q_{\text {set2 }}$ was $<5 \mathrm{cM}$ (as calculated by the $Q T L$ mapping procedures) at a heritability rate of $>50 \%$. The four QTL mapping procedures displayed variation ranges of $<10 \mathrm{cM}$ for the $Q_{\text {set } 1}, Q_{\text {set2 }}$, and $Q_{\text {set } 3}$ positions at heritability rates $>70 \%$. On the other hand, the MQM, ICIM, and MIMR QTL mapping procedures generated variation ranges $<5$ cM for the $Q_{\text {set1 }}, Q_{\text {set2 }}$, and $Q_{\text {set3 }}$ positions.

We observed an increase in the accuracy of QTL mapping and QTL detection power, and a decrease in the variation range of the QTL position with the increase in heritability. In addition, we identified an irregular number of false-positive QTLs. The QTL mapping analysis is of no 
significance at heritability rates $<10 \%$. In a practical study, however, at least three strategies must be applied at QTL heritability rates $<30 \%$ to increase the confidence of the results of QTL mapping: use of multiple QTL mapping strategies (Su et al., 2013), the development of a secondary population based on the results of primary mapping, and the use of repetitive results obtained from experiments conducted over many years or locations. It has been suggested that QTL mapping has meaning at heritability rates $>30 \%$ (at least $>10 \%$ ) in practical marker-assisted breeding.

\section{DISCUSSION}

The QTL analysis was performed using four different QTL mapping procedures (MQM, ICIM, CIM, and MIMR) to increase the persuasiveness and reproducibility of the five sets of computer simulated RIL data. Among these, MQM and CIM could only detect the additive and dominant effects, while ICIM and MIMR could be applied to estimate the additive, dominant, and epistatic effects. The obtained results, which shared some similar aspects with the results of a previous study (Su et al., 2010b and 2013), revealed the importance of selecting the appropriate QTL mapping procedure for different populations, using different genetic models. Many research groups have previously analyzed the QTL mapping accuracy. However, these studies were inadequate; for example, Xu (1994) analyzed only one QTL, linked to a single molecular marker with a heritability of $60 \%$, in order to determine the influence of the QTL mapping accuracy based on the differences in the sample size. On the other hand, Beavis $(1994,1998)$ subjected only one marker interval, located in a single linkage, to computer simulation. A majority of researchers have considered only one QTL in their studies. The genetic parameters of the simulated QTL contains only the additive and dominant effects (always); very few studies have considered any epistatic effects of the QTLs. A majority of the previously conducted studies applied simple genetic statistical models, such as interval mapping or composite interval mapping. The above-mentioned shortcomings were overcome in this study by simulating three additive effects $a_{1}, a_{2}$, and $a_{3}$ and one epistatic effect $a a_{12}$ of $Q_{\text {set1 }}, Q_{\text {set2 }}, Q_{\text {set3 }}$, and the interaction between $Q_{\text {set1 }}$ and $Q_{\text {set2. }}$. The influence of different heritability levels on QTL mapping accuracy was also analyzed using different procedures. The heritability, power of QTL detection, false positive rate, and accuracy of a majority of the QTLs is always between 10 and $30 \%$ in the factual QTL mapping process. The mechanism with which the change in heritability from 10 to $30 \%$ results in changes in the genetic parameters requires further analysis. The results of this study indicated that the QTL mapping accuracy was affected by the heritability level. The results of this study could lay the foundation for the application of markerassisted breeding for some important agronomic traits in plants in the future.

\section{Conflicts of interest}

The authors declare no conflict of interest.

\section{ACKNOWLEDGMENTS}

Research supported by the National Natural Science Foundation of China (Grant \#31460359), the Natural Science Foundation of Guizhou Province of China (Grant \#Qian Kehe J word [2014]2155), the Research Fund for the Doctoral Program of Higher Education of China (Grant \#LPSSYKYJJ201401), and the Fund Project of the Guizhou Provincial Education Department of China (Grant \#Qian jiaohe KY word [2014]235). 


\section{REFERENCES}

Basten CJ, Weir BS and Zeng ZB (1994). Zmap-a QTL cartographer. In: Proceedings of the 5th world congress on genetics applied to livestock production: computing strategies and software (Smith C, Gavora JS, Benkel B, Chesnais J, Fairfull W, Gibson JP, Kennedy BW and Burnside EB, eds.). The organizing committee, 5th world congress on genetics applied to livestock production, Guelph, Ontario, Canada 22: 65-66.

Beavis WD (1994). The power and deceit of QTL experiments. Proceedings of the 49th annual corn and sorghum research conference. American seed trade association, Washington DC, 250-266.

Beavis WD (1998). QTL analysis: power, precision and accuracy. In: Molecular dissection of complex traits (Patterson AH, eds.). CRC Press, Boca Raton, New York, 145-162.

$\mathrm{He}$ XH, Xu CW, Kuai JM, Gu SL, et al. (2001). Principal factors affecting the power of detection and accuracy of QTL mapping. Acta Agron. Sin. 27: 469-475.

Hu ZL, Fritze R and Reecy JM (2007). AnimalQTLdb: a livestock QTL database tool set for positional QTL information mining and beyond. Nucleic Acids Res. 35: 604-609.

Kao CH, Zeng ZB and Teasdale RD (1999). Multiple interval mapping for quantitative trait loci. Genetics 152: 1203-1216.

Lander ES and Bostein DR (1989). Mapping Mendelian factors underlying quantitative traits using RFLP linkage map. Genetics 121: 185-189.

$\mathrm{Li} \mathrm{H,} \mathrm{Ye} \mathrm{G} \mathrm{and} \mathrm{Wang} \mathrm{J} \mathrm{(2007).} \mathrm{A} \mathrm{modified} \mathrm{algorithm} \mathrm{for} \mathrm{the} \mathrm{improvement} \mathrm{of} \mathrm{composite} \mathrm{interval} \mathrm{mapping.} \mathrm{Genetics} \mathrm{175:} \mathrm{361-374.}$

Lu YY and Liu BH (1995). A new computer package for genomic research: PGRI (plant genome research initiative). Abstract submitted to plant genome III conference, San Diego, CA (http://www.intl-pag.org/3/abstracts/201pg3. html).

Manly KF, Cudmore JR and Meer JM (2001). Map Manager QTX, cross-platform software for genetic mapping. Mamm. Genome 12: 930-932.

Moreno-Gonzalez J (1993). Efficiency of generations for estimating marker-associated QTL effects by multiple regression. Genetics 135: 223-231.

Nelson JC (1997). QGENE: Software for marker-based genomic analysis and breeding. Mol. Breed. 3: 239-245.

Su CF, Lu WG, Zhao TJ and Gai JY (2010a). Verification and fine-mapping of QTLs conferring days to flowering in soybean using residual heterozygous lines. Chin. Sci. Bull. 55: 499-508.

Su CF, Zhao TJ and Gai JY (2010b). Simulation comparisons of effectiveness among QTL mapping procedures of different statistical genetic models. Acta Agron. Sin. 36: 1100-1107.

Su CF, Qiu XM, and Ji ZX (2013). Study of strategies for selecting quantitative trait locus mapping procedures by computer simulation. Mol. Breeding 31: 947-956.

Utz HF and Melchinger AE (1996). PLABQTL: a program for composite interval mapping of QTL. J. Agric. Genomics 2: 1-5.

Van Ooijen JW (1992). Accuracy of mapping quantitative trait loci in autogamous species. Theor. Appl. Genet. 84: 803-811.

Van Ooijen JW and Maliepaard C (1996). MapQTL version 3.0: Software for the calculation of QTL positions on genetic maps. Abstract submitted to plant genome IV conference, January 14-18, 1996 San Diego.

Van Ooijen JW, Kyazma BV (2004). MapQTL 5, software for the mapping of quantitative trait loci in experimental populations. Wageningen, The Netherlands

Wang S, Basten CJ and Zeng ZB (2007). Windows QTL cartographer 2.5. Department of Statistics, North Carolina State University, Raleigh, NC. http://statgen.ncsu.edu/qtlcart/WQTLCart.htm.

Wang DL, Zhu J, Li ZK and Paterson AH (2003). User manual for QTLMapper version 1.6-a computer software for mapping quantitative trait loci (QTLs) with main effects, epistatic effects and QTL x environment interactions. Department of Agronomy, Zhejiang University, Hangzhou, China (http://ibi.zju. edu.cn/ software/qtlmapper/).

Wang JK (2009). Inclusive composite interval mapping of quantitative trait genes. Acta Agron. Sin. 35: 239-245.

Xu YB (1994). Factors influencing the power of QTL mapping: Population size. Yi Chuan Xue Bao 20: 537-578.

Yang J, Zhu J and Williams RW (2007). Mapping the genetic architecture of complex traits in experimental populations. Bioinformatics 23: 1527-1536.

Yang J, Hu CC, Hu H, Yu RD, et al. (2008). QTLNetwork: Mapping and visualizing genetic architecture of complex traits in experimental populations. Bioinformatics 24: 721-723. 